\title{
CALCULATIONS OF POINT DEFECTS IN AIN AND GaN; LATTICE RELAXATION EFFECTS
}

\section{GORCZYCA}

High Pressure Research Center PAN, Sokołowska 29, 01-142 Warsaw, Poland

\author{
N.E. Christensen and A. Svane
}

Institute of Physics and Astronomy, University of Aarhus, 8000 Aarhus C, Denmark

Native defects (vacancies, antisites and interstitials) and substitutional impurities ( $\mathrm{Mg}, \mathrm{Zn}$, and $\mathrm{C}$ ) in cubic $\mathrm{GaN}$ and $\mathrm{AlN}$ are studied by means of $a b$ initio theoretical calculations. We examine the energetic positions of the defect levels and lattice relaxations effects. Whereas small relaxations are found in the case of vacancies, the calculations predict that large atomic displacements are associated with antisites. We also discuss the metastable behavior of the nitrogen antisite.

PACS numbers: 73.61.Ey, 71.55.Eq

\section{Introduction}

To study point defects in GaN and Al Nwe have performed first-principles calculations based on the density-functional theory (DFT), using two methods. The first one is the linear muffin-tin orbital (LMTO) [1] Green-function (GF) method [2]. This method ensures that defects are ideal substitutional, i.e., no relaxation of the neighboring atoms is allowed. The results are compared to the calculations using supercell approach and the full-potential [3] LMTO (the second method) with lattice relaxation included. In both methods we are using the local-density approximation LDA [4] to the density-functional theory, in which exchange and correlation effects are accounted for by a simple local potential. In the LMTO GF method the valence electronic structure of the impurity atom is obtained from Green's function $G$, which is found by solving the Dyson equation: $G=G_{0}+G_{0} \Delta V$ with $G_{0}-$ Green's function of the pure crystal host, and $\Delta V$ - the perturbation due to the impurity. The host Green function is calculated from the band structure of the pure crystal within the atomic sphere approximation (ASA), i.e., the crystal volume is approximated by slightly overlapping atom centered spheres, inside which the potential is made spherically symmetric. We are not able to include lattice relaxation in the GF calculation, so in order to study such structural effects we resort to the supercellmethod. Relaxations cannot 
be predicted from total-energy calculations using the ASA. The full nonspherical shapes of potentials and charge distributions must be taken into account. We do this by combining the supercell approach with the full-potential version [3] of the LMTO scheme. Calculations are performed using 32-atom supercells. In contrast to the LMTO GF method where defect states are characterized by a single energy level, in supercell approach the impurity levels in the band gap are determined as the center-of-gravity of the impurity bands.

Both GaN and AlN can crystallize in two phases, the cubic (zinc-blende) and hexagonal (wurtzite) phases. We study here the cubic phase, believing that there is little difference in impurity level positions of the two phases.

\section{Native defect levels}

The energy gap of AlN in the zinc-blende structure is $6.1 \mathrm{eV}$, almost twice of that of $\mathrm{GaN}(3.25 \mathrm{eV})$. Comparing $\mathrm{GaN}$ and $\mathrm{AlN}$, the same sequence of states is seen for all the native defects. The positions of the states in the gap are also similar, with the general rule that they are lying somewhat deeper in the energy gap in AIN. In particular, some states which are resonant with the conduction or valence band in $\mathrm{GaN}$ (as $\mathrm{V}_{\mathrm{N}}$ ) have in AlN moved into the band gap.

Cation vacancies, both in GaN and AlN, create several acceptor states ranging from $0.2 \mathrm{eV}$ to $1.9 \mathrm{eV}$, whereas nitrogen vacancies act as shallow donors. $V_{\text {Cat }}$ in the neutral charge state is a triple acceptor, with a level close to the valence band, which can be filled with three more electrons. Its level position in GaN is $0.2 \mathrm{eV}$ above valence band top, and $0.5 \mathrm{eV}$ in $\mathrm{AlN}$.

As the energy level positions for cation vacancies are very similar in GaN and $A I N$, in the case of nitrogen vacancy the situation is slightly different. In GaN the $a_{1}$ ( $s$-like) state of $\mathrm{V}_{\mathrm{N}}$ is a resonance below the valence band edge, whereas the $t_{2}$ ( $p$-like) state forms a resonance in the conduction band. For the neutral charge state one electron is bound by the positive potential in a shallow donor level characterized by the effective mass of the conduction band minimum, i.e., the nitrogen vacancy acts as a single donor. Nitrogen vacancy states, resonant with the conduction or valence band in GaN, in AlN are moved into the band gap. As results from our calculations the $a_{1}$ (s-like) state of $V_{N}$ lies at $1.7 \mathrm{eV}$ above the valence edge, whereas the $t_{2}$ ( $p$-like) state lies at $0.4 \mathrm{eV}$ below the conduction band edge.

Antisite defects introduce deep levels both in GaN and AlN.

Cation antisite in the neutral charge state is a double acceptor. In GaN its level is situated at about $1.0 \mathrm{eV}$ above the valence band edge, which is a little lower than the position of the same state obtained by the pseudopotential calculations $(\approx 1.5 \mathrm{eV})$ [5]. In AlN the same defect introduces an energy level close to the midgap (about $3 \mathrm{eV}$ above the valence band edge).

Nitrogen antisite behaves almost identically in GaN and AlN. The neutral nitrogen antisite defect, $\mathrm{N}_{\mathrm{Ga}}$, introduces a doubly occupied $a_{1}$ state just above the valence band maximum and an empty triplet close to the conduction band edge.

In zinc-blende crystals there are two interstitial sites with tetrahedral symmetry, surrounded by four cations (i1) or four anions (i2), respectively. We have found that the defect states corresponding to positions $(i 1)$ and $(i 2)$ of an intersti- 
tial gallium atom are very close, giving rise to a deep $s$-state at $1.8 \mathrm{eV}$ and $1.9 \mathrm{eV}$ above the valence band edge, respectively. For the $\mathrm{N}$ interstitial the $s$-states are resonant with the valence band, whereas $t_{2}$ states, occupied by three electrons, are lying at $1.4 \mathrm{eV}$ and $1.9 \mathrm{eV}$ above the valence band maximum, for positions (i1) and $(i 2)$, respectively.

\section{Impurity levels}

In the present work we study the substitutional $\mathrm{C}, \mathrm{Zn}$ and $\mathrm{Mg}$ impurities. Carbon, being a group-IV atom, is an amphoteric impurity in both GaN and AlN. $\mathrm{C}_{\mathrm{N}}$ is a shallow acceptor; it introduces a set of impurity-induced levels, the lowest one (corresponding to neutral charge state) is located about $0.1 \mathrm{eV}$ above the top of valence band in $\mathrm{GaN}$, and a little higher in AlN (about $0.3 \mathrm{eV}$ above valence band edge). $\mathrm{C}_{\mathrm{Cat}}$ introduces an resonant state above the bottom of the conduction band in GaN and effective-mass donor level nearly degenerate with the conduction band in AlN.

A substitutional $\mathrm{Zn}$ and $\mathrm{Mg}$ atoms on a cation site form shallow acceptor states. Our calculated level position for $\mathrm{Zn}$ impurity in $\mathrm{GaN}$ is $0.1 \mathrm{eV}$ above the valence band edge for the neutral state, and $0.2 \mathrm{eV}$ above the valence band edge for AlN. Mg creates even shallower acceptor state. As results from our calculations, in $\mathrm{GaN}$ it is close to the valence band edge for the neutral charge state, in AlN lies about $0.1 \mathrm{eV}$ above the valence band top.

\section{Lattice relaxation effects}

The outward relaxation is found for cation vacancies; less pronounced in GaN - the nearest neighbors are shifted away by $4 \%$ of the bond length, decidedly larger in AlN - $11 \%$ of the bond length. The above results are in perfect agreement with the other theoretical results: the same values were obtained by Neugebauer and Van de Walle [6] for GaN, and by Mattila et al. [7] for AlN.

Lattice relaxation is not substantial in the case of nitrogen vacancy, both in $\mathrm{GaN}$ and $\mathrm{AlN}$. In GaN we found that the nearest neighbor $\mathrm{Ga}$ atoms relax inward by $4 \%$ of the bond length. This is in agreement with pseudopotential calculations [8] (inward relaxation equal about $3 \%$ of the bond length). In AlN we have not found any relaxation connected with nitrogen vacancy. For both compounds we have found an outward relaxation around the cation antisite. In GaN the distance to the nearest neighbors is increased by $12 \%$ of the ideal bond length, in AlN by $18 \%$. The result for $\mathrm{GaN}$ is in agreement with the pseudopotential calculations [5] (11\% outward relaxation).

Assuming for nitrogen antisite the ideal symmetry, we find a small, $1 \%$, outward displacement of the nearest neighbor nitrogen atoms. Allowing symmetry changes, we find a large shift of the nitrogen antisite along the threefold symmetry axis. In the case of $\mathrm{N}_{\mathrm{Ga}}$ the bond distance to the nearest neighbor atom is reduced by $30 \%$ (in agreement with pseudopotential calculations [5]). Supercell calculations for $\mathrm{N}_{\mathrm{Al}}$ yield similar result - reduction of the bond length to the nearest nitrogen atom by $29 \%$, which agrees with the result obtained by Mattila et al. [7].

We investigate also the metastable behavior of the nitrogen antisite reported already by Mattila et al. [7]. When the antisite is displaced along the (111) axis 
away from the nearest neighbor atom a second energy minimum occurs. We find it at a displacement equal to $49 \%$ of the bond length for AlN and $45 \%$ for GaN. The relaxation of the remaining atoms at the displaced site is spherically symmetric - the three atoms surrounding the antisite relax inwards. In AIN by $8 \%$ of the bond length, in $\mathrm{GaN}$ by $12 \%$ of the bond length. The above results are in good agreement with the pseudopotential calculation by Mattila et al. [7].

When atoms around the carbon impurity on nitrogen site are allowed to relax the small inward relaxation is found in the case of GaN ( $4 \%$ of the bond length). There is no relaxation in the case of AlN. The above results differ somehow from those obtained by pseudopotential method [9] where a small inward relaxation is found for $\mathrm{GaN}$ ( $2 \%$ of the bond length), and a small outward relaxation for AlN (also $2 \%$ ). The relaxation around carbon atom at cation site is large, and it is accompanied by the reduction of the nearest neighbor bond length by about $18 \%$ in GaN and about $17 \%$ in $\mathrm{AIN}$.

Considering the lattice relaxation effects connected with $\mathrm{Mg}$ and $\mathrm{Zn}$ impurities, in both cases we have found an outward relaxation around the defect. It is smaller in $\mathrm{GaN}$ ( $2 \%$ and $4 \%$ of the bond length for $\mathrm{Mg}$ and $\mathrm{Zn}$, respectively), and greater in AlN (7\% and 10\%).

\section{Summary}

In conclusion, we have calculated the energy positions of vacancies and antisites together with some common dopants in GaN and AlN, including lattice relaxation effects.

We find pronounced outward lattice relaxation around cation antisites. In the more complex case like nitrogen antisite, which seems to be similar to the EL2 in GaAs, large inward relaxation is found. The distance from antisite to the nearest neighbor atom is reduced by approximately $30 \%$ and becomes comparable to the $\mathrm{N}_{2}$ dimer bond length. Pronounced inward relaxation (about $18 \%$ of the bond length) is found also for $\mathrm{C}$ impurity in cation site. In remained cases relaxation is rather small or negligible. Exceptions here are the $\mathrm{V}_{\mathrm{Al}}$ and $\mathrm{Zn}_{\mathrm{Al}}$ (outward relaxation about $10 \%$ of the $i d e a l$ bond length).

\section{Acknowledgment}

This work was partially supported by the Committee for Scientific Research grant No. 2P03B 17810.

\section{References}

[1] O.K. Andersen, Phys. Rev. B 12, 3060 (1975).

[2] O. Gunnarsson, O. Jepsen, O.K. Andersen, Phys. Rev. B 27, 7144 (1983).

[3] M. Methfessel, Phys. Rev. B 38, 1537 (1988).

[4] R.O. Jones, O. Gunnarsson, Rev. Mod. Phys. 61, 681 (1989).

[5] P. Bogusławski, E.L. Briggs, J. Bernholc, Phys. Rev. B 51, 17255 (1995).

[6] J. Neugebauer, C.G. Van de Walle, Phys. Rev. B 50, 8067 (1994).

[7] T. Mattila, A.P. Seitsonen, R.M. Nieminen, Phys. Rev. B 54, 1454 (1996).

[8] J. Neugebauer, C.G. Van de Walle, Mater. Res. Soc. Symp. Proc. 339, 687 (1994).

[9] P. Boguslawski, J. Bernholc, Acta Phys. Pol. A 90, 735 (1996). 